\title{
Quantization of Contact Manifolds
}

By

\author{
Masaki KASHIWARA*
}

\begin{abstract}
We show the existence of the stack of micro-differential modules on an arbitrary contact manifold, although we cannot expect the global existence of the ring of micro-differential operators.
\end{abstract}

\section{§ 0. Inroduction}

In $[\mathrm{SKK}]$, we defined the sheaf of micro-differential operators on the cotangent bundle and we associated a quantized contact transformation with a given contact transformation.

More precisely, for a complex manifold $X$, let us denote by $\mathscr{E}_{X}$ the ring of micro-differential operators regarded as a sheaf of rings on the projective cotangent bundle $P^{*} X$. Let $X$ and $Y$ be two manifolds with the same dimension. Let $U_{X}$ and $U_{Y}$ be open subsets of $P^{*} X$ and $P^{*} Y$ respectively, and let $f: U_{X} \rightarrow U_{Y}$ be a holomorphic map preserving the canonical 1-form. Then for any point $p \in U_{X}$ there exists an open neighborhood $U$ of $p$ and a C-ring isomorphism $\varphi$ : $\left.\left.f^{-1} \mathscr{E}_{Y}\right|_{U} \rightarrow \mathscr{E}_{X}\right|_{U}$. Such a $\varphi$ is not unique, although with other extra data we can reduce the uniqueness of $\varphi$ up to the inner automorphism by micro-differential operators with 1 as its principal symbol.

Now let us consider a contact manifold $Z$ with $(2 n+1)$ dimension. This means that $Z$ is endowed with an invertible $\mathscr{O}_{Z}$-module $\mathcal{O}_{Z}(1)$ and a 1 -form $\omega \in$ $\Gamma\left(Z, \Omega_{Z}^{1} \otimes \mathcal{O}_{Z}(1)\right)$ such that $\omega \wedge(d \omega)^{n}$ is a generator of $\Omega_{Z}^{2 n+1} \otimes \mathcal{O}_{Z}(2 n+1)$. Here $\mathscr{O}_{Z}(k)=\mathscr{O}_{Z}(1)^{\otimes k}$.

The purpose of this paper is to show that we can naturally define a stack (a sheaf of categories) on $Z$ that is locally isomorphic to the stack of modules over the ring of micro-differential operators.

Let us take an open covering $Z=\bigcup_{i \in I} U_{i}$ and contact embeddings $f_{i}: U_{i} \hookrightarrow$ $P^{*} X_{i}$. Set $\mathscr{A}_{i}=f_{i}^{-1}\left(\left(\Omega_{X_{2}}^{n}\right)^{\otimes 1 / 2} \otimes \mathscr{E}_{X_{l}} \otimes\left(\Omega_{X_{i}}^{n}\right)^{\otimes-1 / 2}\right)$. Then, $\mathscr{A}_{i}$ is a sheaf of C-rings on

Received February 13, 1992.

1991 Mathematics Subject Classification (s): 53C99

* RIMS, Kyoto University, Sakyo-ku, 606-01, JAPAN. 
$U_{\imath}$ endowed with an antiautomorphism $*$ such that $*^{2}=1$. The ring $\mathscr{A}_{\imath}$ has the order filtration $F\left(\mathscr{A}_{i}\right)$ such that $G r_{k}^{F} \mathscr{A}_{2}=\mathscr{O}_{Z}(k)$.

Lemma 1. Let $\mathscr{G}$ be the sheaf of automorphisms of $\mathscr{A}_{1}$ commuting with *. Then $\left\{P \in F_{0}\left(\mathscr{A}_{2}\right) ; P^{*} P=1, \sigma_{0}(P)=1\right\} \rightarrow \mathscr{G}$ given by $P \mapsto \operatorname{Ad}(P)$ is bijective. Here $\sigma_{0}$ is the symbol map $F_{0}\left(\mathscr{A}_{2}\right) \rightarrow G r_{0}^{F} \mathscr{A}_{\imath}=\mathcal{O}_{Z}$.

Proof. For $\lambda \in \mathbf{C}$, let $\mathscr{E}(\lambda)$ be the sheaf of micro-differential operators of order $\lambda+\mathbf{Z}_{\leq 0}$. Then any automorphism $\varphi$ of $\mathscr{E}_{X}$ is given by $\operatorname{Ad}(P)$ for some $\lambda$ and some invertible element $P$ of $\mathscr{E}(\lambda)$. If $\varphi$ commutes with *, then $\operatorname{Ad}\left(P^{*} P\right)=$ id and hence $P^{*} P$ must be constant. Hence $P$ is order 0 and we can normalize $P^{*} P=1$ and $\sigma_{0}(P)=1$ by dividing $P$ by a suitable constant.

Now, shrinking $U_{\imath}$ if necessary, we may assume that there exists a C-ring isomorphism $f_{i j}:\left.\left.\mathscr{A}_{j}\right|_{U_{\imath}} \stackrel{\sim}{\rightarrow} \mathscr{A}_{\imath}\right|_{U_{\imath}}$, which commutes with *. Here we employed the notation

$$
U_{\imath_{0} \imath_{1} \cdots i_{p}}=U_{\imath_{0}} \cap U_{i_{1}} \cap \cdots \cap U_{i_{p}}
$$

Then $f_{i j} \circ f_{j k}:\left.\left.\mathscr{A}_{k}\right|_{U_{\imath \jmath k}} \rightarrow \mathscr{A}_{i}\right|_{U_{\imath, k}}$ is not equal to $\left.f_{\imath k}\right|_{U_{i k}}$ in general. Hence we cannot patch $\mathscr{A}_{i}$ together to get a ring globally defined on $Z$.

By Lemma 1, there exists $P_{\imath \jmath k} \in \Gamma\left(U_{\imath \jmath k} ; F_{0}\left(\mathscr{A}_{\imath}\right)\right)$ such that

$$
f_{\imath \jmath} \circ f_{\jmath k}=A d\left(P_{\imath \jmath k}\right) \circ f_{i k} \text { and } P_{i \jmath k}^{*} P_{\imath \jmath k}=1, \sigma_{0}\left(P_{\imath \jmath k}\right)=1 \text {. }
$$

For $i, j, k, l \in I$, we have

$$
\left(f_{\imath \jmath} \circ f_{j k}\right) \circ f_{k l}=A d\left(P_{\imath \jmath k}\right) \circ f_{\imath k} \circ f_{k l}=A d\left(P_{\imath \jmath k} P_{k l}\right) \circ f_{\imath l}
$$

and

$f_{i \jmath} \circ\left(f_{\jmath k} \circ f_{k l}\right)=f_{i \jmath} \circ \operatorname{Ad}\left(P_{\jmath k l}\right) \circ f_{\jmath l}=A d\left(f_{\imath \jmath}\left(P_{j k l}\right)\right) \circ f_{\imath \jmath} \circ f_{\jmath l}=\operatorname{Ad}\left(f_{\imath \jmath}\left(P_{\jmath k l}\right) P_{\imath \jmath l}\right) \circ f_{\imath l}$.

Hence by Lemma 1, we obtain

$$
P_{\imath \jmath k} P_{\imath k l}=f_{i \jmath}\left(P_{j k l}\right) P_{\imath \jmath l} .
$$

This cocycle relation permits us to patch the categories of $\mathscr{A}_{\imath}$-modules to get a stack globally defined over $Z$.

\section{§ 1. Stack}

Let us recall the definition of a stack on a topological space $X$. A prestack $\mathscr{C}$ on $X$ consists of following data:

(1.1) a category $\mathscr{C}(U)$ for any open subset $U$ of $X$, 
(1.2) A functor $r_{V U}: \mathscr{C}(U) \rightarrow \mathscr{C}(V)$ for open subsets $V$ and $U$ with $V \subset U$.

(1.3) An isomorphism of functors $\theta_{W V U}: r_{W V} \circ r_{V U} \rightarrow r_{W U}$ for open subsets $W \subset V \subset U$.

They are assumed to satisfy the following axioms.

(PS1) $\quad r_{U U}=i d$

(PS2) $\theta_{U U U}=i d$.

(PS3) For open subsets $U_{1} \subset U_{2} \subset U_{3} \subset U_{4}$, the diagram

$$
\begin{array}{rr}
r_{U_{1} U_{2}} \circ r_{U_{2} U_{3}} \circ r_{U_{3} U_{4}} \stackrel{\stackrel{\theta_{U_{2} U_{3} U_{4}}}{\longrightarrow}}{\longrightarrow} r_{U_{1} U_{2}} \circ r_{U_{2} U_{4}} \\
\downarrow \theta_{U_{1} U_{2} U_{3}} & \downarrow \theta_{U_{1} U_{2} U_{4}} \\
r_{U_{1} U_{3}} \circ r_{U_{3} U_{4}} \stackrel{\theta_{U_{1} U_{3} U_{4}}}{\longrightarrow} & r_{U_{1} U_{4}}
\end{array}
$$

commutes.

A prestack $\mathscr{C}$ is called a stack if it satisfies furthermore the following axioms.

(S1) For any open subset $U$ and $A, B \in O b(\mathscr{C}(U))$, the presheaf on $U$

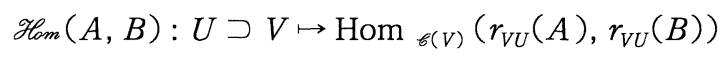

is a sheaf.

(S2) Let $\left\{U_{\imath}\right)$ be an open covering of an open set $U, A_{\imath} \in O b\left(\mathscr{C}\left(U_{\imath}\right)\right)$ and let $\varphi_{\imath \jmath}: r_{U_{\imath}, U_{j}}\left(A_{\jmath}\right) \rightarrow r_{U_{\imath}, U_{l}}\left(A_{2}\right)$ be an isomorphism. Assume the commutativity of the following diagram for any $i, j, k$ :

$$
\begin{aligned}
& r_{U_{\imath \jmath} U_{j k}} r_{U_{j k} U_{k}} A_{k} \stackrel{\varphi_{j k}}{\longrightarrow} r_{U_{\imath \jmath k} U_{j k}} r_{U_{j k} U_{j}} A_{\jmath} \stackrel{\theta_{U_{1, k} U_{k} U_{j}}}{\longrightarrow} \quad r_{U_{\imath, k} U_{j}} A_{\jmath} \\
& \downarrow \theta_{U_{1, k}, U_{j}, U_{k}} \quad \imath \uparrow \theta_{U_{u, k} U_{U,} U_{j}} \\
& r_{U_{\imath \jmath} U_{k}} A_{k} \quad r_{U_{\imath \jmath k} U_{\imath}}, r_{U_{\imath}, U_{j}} A_{\text {j }} \\
& \text {, } \uparrow_{\theta_{U_{i, k} U_{i k} U_{k}}} \quad \downarrow \varphi_{1} \\
& r_{U_{\imath \jmath k} U_{\imath k}} r_{U_{\imath k} U_{k}} A_{k} \quad r_{U_{\imath \jmath k} U_{l}} r_{U_{\imath}, U_{l}} A_{\imath} \\
& \downarrow \varphi_{1 k} \\
& r_{U_{\imath, k} U_{\imath k}} r_{U_{\imath k} U_{l}} A_{\imath} \\
& \stackrel{\theta_{U_{l, k} U_{1 k} U_{i}}}{\longrightarrow} \\
& \downarrow \theta_{U_{1, k} U_{1}, U_{\mathrm{t}}} \\
& r_{U_{\imath, k} U_{l}} A_{\imath}
\end{aligned}
$$

Then there exist an object $A$ of $\mathscr{C}(U)$ and a family of isomorphisms $\psi_{\imath}: r_{U_{\imath} U}(A) \stackrel{\sim}{\rightarrow} A_{\imath}$ such that 


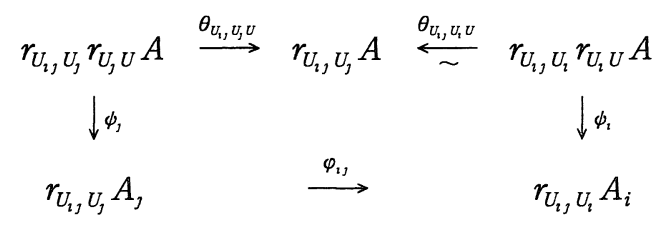

commutes.

For an open subset $U$ of $X$, we can define the restriction $\left.\mathscr{C}\right|_{U}$ to $U$, which is a stack on $U$.

For two stacks $\mathscr{C}_{1}, \mathscr{C}_{2}$ on $X$, we can define the notion of functors from $\mathscr{C}_{1}$ to $\mathscr{C}_{2}$ and for two functors $f, g$ from $\mathscr{C}_{1}$ to $\mathscr{C}_{2}$, we can define the notion of morphisms from $f$ to $g$. We call a functor $f: \mathscr{C}_{1} \rightarrow \mathscr{C}_{2}$ an equivalence if there exists a functor $g: \mathscr{C}_{2} \rightarrow \mathscr{C}_{1}$ such that $f \circ g$ and $g \circ f$ are isomorphic to the identity respectively.

\section{§ 2. Patching of Stacks}

Let $\left\{U_{i}\right\}$ be an open covering of $X$ and $\mathscr{C}_{i}$ a stack on $U_{i}$. Let $\varphi_{i j}:\left.\mathscr{C}_{\imath}\right|_{U_{\imath}} \rightarrow$ $\left.\mathscr{C}_{i}\right|_{U_{\imath}}$, be an equivalence of stacks. Let $\phi_{i j k}: \varphi_{i j} \circ \varphi_{j k} \stackrel{\sim}{\rightarrow} \varphi_{i k}$ be an isomorphism of functors from $\left.\mathscr{C}_{k}\right|_{U_{\imath \jmath k}}$ to $\left.\mathscr{C}_{i}\right|_{U_{\imath \jmath k}}$. Assume that

For any $i, j, k, l$ the diagram

$(P C)$

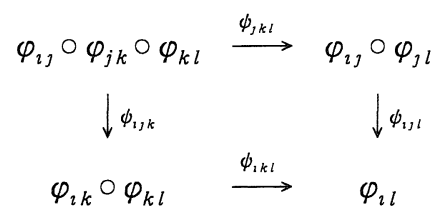

commutes.

Then there exists a stack $\mathscr{C}$ and an equivalence $\left.\mathscr{C}\right|_{U_{\imath}} \rightarrow \mathscr{C}_{\imath}$ satisfying the plausible compatibility conditions. Such a $\mathscr{C}$ is unique up to equivalence.

\section{§ 3. Patching of Stacks of Modules}

In this paper, a ring means a (not necessarily commutative) ring with 1 . Let $\left\{U_{i}\right\}$ be an open covering of $X$ and let $\mathscr{A}_{i}$ be a sheaf of rings on $U_{i}$. Assume that there is given a ring isomorphism $f_{i j}:\left.\left.\mathscr{A}_{j}\right|_{U_{\imath j}} \rightarrow \mathscr{A}_{i}\right|_{U_{\imath}}$ and $a_{i j k} \in \Gamma\left(U_{\imath \jmath k} ; \mathscr{A}_{\imath}^{\times}\right)$such that

$$
f_{i \jmath} \circ f_{j k}=A d\left(a_{\imath \jmath k}\right) f_{i k} \quad \text { in } \operatorname{Hom}\left(\left.\mathscr{A}_{k}\right|_{U_{\imath, k},},\left.\mathscr{A}_{i}\right|_{U_{\imath \jmath k}}\right)
$$

and 


$$
a_{i j k} a_{i k l}=f_{i j}\left(a_{j k l}\right) a_{i j l} \text { in } \Gamma\left(U_{i j k} ; \mathscr{A}_{i}^{\times}\right)
$$

Here $\mathscr{A}_{i}{ }^{\times}$denotes the sheaf of invertible sections of $\mathscr{A}_{i}$.

Note that if the $\mathscr{A}_{i}$ are commutative, then $\left\{f_{i j}\right\}$ satisfies the chain conditions and hence we can define the globally defined ring $\mathscr{A}$ such that $\left.\mathscr{A}\right|_{U_{2}} \simeq \mathscr{A}_{i}$. In a noncommutative case, we cannot construct such an $\mathscr{A}$ in general, but we can construct a stack locally isomorphic to the stack of $\mathscr{A}_{i}$-modules.

Let $\operatorname{Mod}\left(\mathscr{A}_{i}\right)$ be the stack of left $\mathscr{A}_{i}$-modules on $U_{i}$. In order to patch $\operatorname{Mod}\left(\mathscr{A}_{i}\right)$ together, let us apply the result in $\S 2$.

For $M \in \operatorname{Mod}\left(\mathscr{A}_{j}\right)$, let $\varphi_{i j}(M)$ be the $\mathscr{A}_{i}$-module with a sheaf isomorphism $\alpha_{i j}: M \rightarrow \varphi_{i j}(M)$ such that

$$
a \alpha_{i j}(u)=\alpha_{i j}\left(f_{j i}(a) u\right) \text { for } a \in \mathscr{A}_{i} \text { and } u \in M .
$$

This defines the functor $\varphi_{i j}:\left.\left.\operatorname{Mod}\left(\mathscr{A}_{j}\right)\right|_{U_{\imath}} \rightarrow \operatorname{Mod}\left(\mathscr{A}_{i}\right)\right|_{U_{\imath}}$.

Let us define an isomorphism of functors

$$
\phi_{i j k}: \varphi_{i j} \circ \varphi_{j k} \rightarrow \varphi_{i k}
$$

as follows. For $\left.M \in \operatorname{Mod}\left(\mathscr{A}_{k}\right)\right|_{U_{\imath \jmath k}}$, we define

$$
\phi_{i j k}(M): \varphi_{i j} \circ \varphi_{j k}(M) \rightarrow \varphi_{i k}(M)
$$

by $\alpha_{i j} \alpha_{j k}(u) \mapsto \alpha_{i k}\left(a_{k j i}^{-1} u\right)$ for $u \in M$. Let us check that $\phi_{i j k}(M)$ is $\mathscr{A}_{i}$-linear. For $a \in \mathscr{A}_{i}$ and $u \in M$, we have

$$
a \alpha_{i j} \alpha_{j k}(u)=\alpha_{i j}\left(f_{j i}(a) \alpha_{j k}(u)\right)=\alpha_{i j} \alpha_{j k}\left(f_{k j} f_{j i}(a) u\right)=\alpha_{i j} \alpha_{j k}\left(\alpha_{k j i} f_{k i}(a) a_{k j i}^{-1} u\right) .
$$

Hence we obtain

$$
\phi_{i j k}(M)\left(a \alpha_{i j} \alpha_{j k}(u)\right)=\alpha_{i k}\left(f_{k i}(a) a_{k \jmath i}^{-1} u\right)=a \alpha_{i k}\left(a_{k j i}^{-1} u\right)=a \phi_{i j k}(M)\left(\alpha_{i j} \alpha_{j k}(u)\right) .
$$

Thus, $\phi_{i j k}(M)$ is $\mathscr{A}_{i}$-linear and hence $\phi_{i j k}$ is a well-defined morphism of functors.

Next, we shall check the chain condition (PC). The composition $\phi_{i k l} \circ \phi_{i j k}$ is calculated as follows:

$$
\begin{aligned}
\phi_{i k l} \phi_{i j k}\left(\alpha_{i j} \alpha_{j k} \alpha_{k l}(u)\right) & =\phi_{i k l} \alpha_{i k}\left(a_{k j i}^{-1} \alpha_{k l}(u)\right)=\phi_{i k l} \alpha_{i k} \alpha_{k l}\left(f_{l k}\left(a_{k j i}^{-1}\right) u\right) \\
& =\alpha_{i l}\left(a_{l k i}^{-1} f_{l k}\left(a_{k j l}^{-1}\right) u\right) .
\end{aligned}
$$

Similarly, we have

$$
\begin{aligned}
\phi_{i j l} \phi_{j k l}\left(\alpha_{i j} \alpha_{j k} \alpha_{k l}(u)\right) & =\phi_{i j l} \alpha_{i j}\left(\phi_{j k l}\left(\alpha_{j k} \alpha_{k l}(u)\right)\right)=\phi_{i j l} \alpha_{i j} \alpha_{j l}\left(a_{l k j}^{-1} u\right) \\
& =\alpha_{i l}\left(a_{l j i}^{-1} a_{l k j}^{-1} u\right) .
\end{aligned}
$$

Then $\phi_{i k l} \circ \phi_{i j k}=\phi_{i j l} \circ \phi_{j k l}$ follows from (C2).

By the arguments in $\S 2$, we can patch $\operatorname{Mod}\left(\mathscr{A}_{i}\right)$ together and we obtain a globally defined stack. 
Now, we can apply this result to the situation in $\S 1$, and we obtain

Theorem 2. For any contact manifold $Z$, we can define canonically a stack $\operatorname{Mod}(Z)$ on $Z$, which is locally equivalent to the stack of $\mathscr{E}_{X}$-modules.

We call an object $L$ of $\operatorname{Mod}(Z)$ invertible if it is locally isomorphic to $\mathscr{A}_{2}$. If there is an invertible object $L$, then $\mathscr{A}=\operatorname{End}(L)$ is a sheaf of rings locally isomorphic to the sheaf of micro-differential operators and $\operatorname{Mod}(Z)$ is equivalent to $\operatorname{Mod}(\mathscr{A})$. Hence the existence of a globally defined ring of micro-differential operators is equivalent to the existence of an invertible object.

\section{§4. Sheaf of Microfunctions}

Let $Z$ be a contact manifold and let $Z_{\mathbf{R}}$ be a real analy tic submanifold such that $Z$ is a complexification of $Z_{\mathrm{R}}$. Let $\bar{Z}$ be the complex conjugate of $Z$. By shrinking $Z$ if necessary, we may assume that there is an isomorphism of complex manifolds $\bar{Z} \rightarrow Z$ that is set-theoretically the identity on $Z_{\mathrm{R}}$. Assume that and $\mathcal{O}_{Z}(1)$ has a complex conjugation and $\sqrt{-1} \omega$ is invariant by the complex conjugation. Let $\Lambda_{x}$ be the set of oriented Lagrangian vector subspaces in $T_{x}\left(Z_{\mathrm{R}}\right)$. Then $\Lambda=\cup \Lambda_{x}$ is a fiber bundle over $Z_{\mathbf{R}}$. Let $\pi: \Lambda \rightarrow Z_{\mathbf{R}}$ be the projection.

Since $\pi_{1}\left(\Lambda_{x}\right) \cong \mathbb{Z}$, there is a canonical double covering $p: \tilde{\Lambda} \rightarrow \Lambda \times{ }_{Z_{\mathrm{R}}} \Lambda$ over $\Lambda \times{ }_{Z_{\mathrm{R}}} \Lambda$ with a canonical map $i: \Lambda \rightarrow \tilde{\Lambda}$ such that $p \circ i$ is the diagonal embedding.

Let $p_{1}$ and $p_{2}$ be the first and the second projection from $\Lambda \times_{Z_{\mathrm{R}}} \Lambda$ onto $\Lambda$. Let $\sigma$ be the covering automorphism of $p: \tilde{\Lambda} \rightarrow \Lambda \times{ }_{Z_{\mathrm{R}}} \Lambda$ and let $L$ be the subsheaf of $p_{*} \mathbb{C}_{\tilde{\Lambda}}$ consisting of sections $s$ such that $\sigma^{*} s=-s$. Then $L$ is locally isomorphic to $\mathbf{C}_{\Lambda \times Z_{\mathrm{R}} \Lambda}$ and $i^{-1} L$ is canonically isomorphic to $\mathbf{C}_{\Lambda}$. Let $\mathscr{C}$ be the stack on $Z_{\mathrm{R}}$ defined by : for any open subset $U$ of $Z_{\mathrm{R}}, \mathscr{C}(U)=\left\{(F, \varphi) ; F\right.$ is a sheaf on $\pi^{-1}(U)$ and $\varphi$ is an automorphism $p_{2}^{-1} F \otimes L \simeq p_{1}^{-1} F$ such that $i^{-1} \varphi: F \rightarrow F$ is equal to the identity\}.

Then $\mathscr{C}$ is a stack locally equivalent to the stack of sheaves on $Z_{\mathrm{R}}$.

We can define the stack $\mathscr{C} \otimes \operatorname{Mod}(Z)$ over $Z_{\mathbf{R}}$ in an obvious way. Then for $M \in \operatorname{Mod}(Z)$ and $F \in \mathscr{C} \otimes \operatorname{Mod}(Z), \mathscr{H}_{0}(M, F)$ belongs to $\mathscr{C}$.

Now, we have

Proposition 3. We can define canonically an object $\mathscr{C}_{Z_{\mathbf{R}}}$ of $\mathscr{C} \otimes \operatorname{Mod}(Z)$, which is locally isomorphic to the sheaf of microfunctions.

\section{§ 5. Regular Holonomic Systems}

Since the notion of regular holonomic $\mathscr{E}$-modules is invariant by the quantized contact transformations, we can define the notion of regular holonomic systems for objects in $\operatorname{Mod}(Z)$. The subcategory $\operatorname{Reg}(Z)$ of regular holonomic 
systems in $\operatorname{Mod}(Z)$ forms a full abelian subcategory of $\operatorname{Mod}(Z)$.

Let $\Lambda$ be a Lagrangian submanifold of $Z$. Then $\left(\Omega_{\Lambda}^{\mathrm{d} m \Lambda}\right)^{\otimes 1 / 2}$ defines the stack $\mathscr{C}_{\Lambda}$ of twisted sheaves (cf. e.g. [K1]). The stack $\mathscr{C}_{\Lambda}$ is locally isomorphic to the stack of sheaves on $\Lambda$ and it contains $\left(\Omega_{\Lambda}^{\operatorname{dim} \Lambda}\right)^{\otimes 1 / 2}$ as an object. Then we have the following proposition, which is a translation of Theorem (10.3) [K2]

Proposition 4. The category of regular holonomic systems with support in $\Lambda$ is equivalent to the category of locally constant objects in $\mathscr{C}_{\Lambda}$.

Here a locally constant object $L$ in $\mathscr{C}_{\Lambda}$ is an object in $\mathscr{C}_{\Lambda}$ locally isomorphic to a constant sheaf of finite rank.

\section{$\S$ 6. Discussion}

We know by the Riemann-Hilbert correspondence, the category of perverse sheaves is equivalent to the category of regular holonomic $D_{X}$-modules. We can ask what is the stack of "perverse sheaves on $Z$ ", which is equivalent to the stack $\operatorname{Reg}(Z)$ of regular holonomic systems on $Z$.

Another question is: we defined $\operatorname{Mod}(Z)$ for a contact manifold $Z$. Is there an analogue of $\operatorname{Mod}(Z)$ on any Poisson manifold $Z$ ?

\section{References}

[G] Giraud, J., Cohomologie non abélienne, Springer, 1971.

[SKK] Sato, M., Kawai T. and Kashiwara, M., Microfunctions and Pseudo-differential Equations, Lecture Notes in Math., Springer, 287 (1973), 265-523.

[K1] Kashiwara, M., Representation theory and $D$-modules on flag variety, Asterisque, 173-174 (1989), 55-110.

[K2] - Introduction to Microlocal Analysis, Enseign. Math., 32 (1986), 227-259.

[SGA1] Séminaire de Géométrie Algébrique du Bois Marie 1960/61 SGA1, Revetements Etales et Group Fondemental, Lecture Notes in Math., Springer, 224 (1971). 
